\title{
Activation of nuclear factor-kappa $B$ by linear ubiquitin chain assembly complex contributes to lung metastasis of osteosarcoma cells
}

\author{
MASATO TOMONAGA ${ }^{1}$, NOBUYUKI HASHIMOTO ${ }^{1}$, FUMINORI TOKUNAGA ${ }^{2}$, \\ MEGUMI ONISHI $^{1}$, AKIRA MYOUI ${ }^{1}$, HIDEKI YOSHIKAWA $^{1}$ and KAZUHIRO IWAI ${ }^{2,3}$ \\ Departments of ${ }^{1}$ Orthopedic Surgery, ${ }^{2}$ Biophysics and Biochemistry, Graduate School of Medicine,${ }^{3}$ Cell Biology \\ and Metabolism Group, Graduate School of Frontier Biosciences, Osaka University, Osaka, Japan
}

Received July 28, 2011; Accepted August 8, 2011

DOI: 10.3892/ijo.2011.1209

\begin{abstract}
NF- $\mathrm{\kappa B}$ is involved in the metastasis of malignant cells. We have shown that NF- $\mathrm{KB}$ activation is involved in the pulmonary metastasis of LM8 cells, a highly metastatic subclone of Dunn murine osteosarcoma cells. Recently, it was determined that a newly identified type of polyubiquitin chain, a linear polyubiquitin chain, which is specifically generated by the linear ubiquitin chain assembly complex (LUBAC), plays a critical role in NF- $\mathrm{kB}$ activation. Here, we have evaluated the roles of LUBAC-mediated NF- $\mathrm{KB}$ activation in the development of lung metastasis of osteosarcoma cells. All three components of LUBAC (HOIL-1L, HOIP and SHARPIN) were highly expressed in LM8 cells compared to Dunn cells. Attenuation of LUBAC expression by stable knockdown of HOIL-1L in LM8 cells significantly suppressed NF- $\kappa B$ activity, invasiveness in vitro and lung metastasis. Induction of intracellular adhesion molecule-1 (ICAM-1) expression by LUBAC is involved in cell retention in the lungs after an intravenous inoculation of tumor cells. Moreover, we found that knockdown of LUBAC decreased not only the number but also the size of the metastatic nodules of LM8 cells in the lungs. These results indicate that LUBAC-mediated NF- $\mathrm{KB}$ activation plays crucial roles in several steps involved in metastasis, including extravasation and growth of osteosarcoma cells in the lung, and that suppression of LUBAC-mediated linear polyubiquitination activity may be a new approach to treat this life-threatening disease of young adolescents.
\end{abstract}

Correspondence to: Dr Masato Tomonaga or Dr Kazuhiro Iwai, Osaka University Graduate School of Medicine, 2-2 Yamada-oka, Suita, Osaka 565-0871, Japan

E-mail: masatotomonaga@ort.med.osaka-u.ac.jp

E-mail:kiwai@cellbio.med.osaka-u.ac.jp

Key words: linear ubiquitin chain assembly complex, nuclear factorkappa B, osteosarcoma, lung metastasis

\section{Introduction}

Osteosarcoma is the most common primary malignant bone tumor that mainly affects adolescents. Fortunately, a multidisciplinary approach including chemotherapy, wide resection surgery and/or radiation therapy has greatly contributed to the successful treatment of this life-threatening disease, especially when it arises in the extremities. Recent studies have reported excellent clinical results with 5-year overall survival rates of $70-80 \%(1,2)$. However, in patients with lung metastasis, which is the most prevalent metastatic site of the sarcoma, the 5-year survival rate remains only $20-30 \%(3,4)$. Thus, lung metastasis of osteosarcoma is still raising challenging questions for both researchers and clinicians, including the mechanism underlying the selective metastasis of this sarcoma to the lungs. These questions must be answered in order to develop successful treatments for metastatic osteosarcoma. Therefore, we have established a mouse model to analyze the molecular events involved in the development of lung metastasis (5).

In 1998, we established a highly metastatic cell line, lung metastasis 8 (LM8), from parental Dunn murine osteosarcoma cells through eight times cycles of in vivo selection according to Fiddler's method (5). Nuclear factor-kappa B (NF- $\mathrm{BB}$ ) was constitutively activated in LM8 cells, and NF- $\mathrm{kB}$-related genes such as matrix metalloproteinase $(M M P)$, vascular endothelial growth factor (VEGF) and intracellular adhesion molecule-1 (ICAM-1) were up-regulated in LM8 cells as compared to the parental Dunn cells (5-7). Since the constitutive activation of $\mathrm{NF}-\mathrm{\kappa B}$ is related to the highly metastatic potential of LM8 cells, we performed a therapeutic experiment using parthenolide (PAL), a natural NF-kB inhibitor obtained from an herbal extract (8). Lung metastasis of LM8 cells was significantly attenuated without affecting the growth of primary tumors when PAL was administered to mice concurrently with tumor inoculation. However, the administration of PAL $48 \mathrm{~h}$ after inoculation did not suppress the lung metastasis of LM8. These results suggest that NF- $\mathrm{kB}$ activity plays a crucial role in the initial colonization of LM8 cells in the lungs.

$\mathrm{NF}-\mathrm{KB}$ is a ubiquitous, dimeric transcription factor composed of the Rel family proteins. In the resting state, NF- $\kappa B$ binds to its inhibitor proteins (inhibitor $\kappa \mathrm{B}$ : $\mathrm{I} \kappa \mathrm{B}$ ) and is confined to the 
cytoplasm of cells. Exposure of cells to inflammatory cytokines, such as tumor necrosis factor $\alpha$ (TNF- $\alpha)$ and interleukin- $1 \kappa$

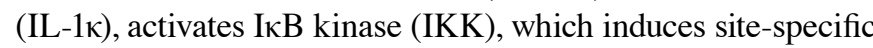
phosphorylation of $\mathrm{I} \kappa \mathrm{B}$ followed by its proteasomal degradation. The liberated $\mathrm{NF}-\kappa \mathrm{B}$ translocates into the nucleus and induces the expression of target genes, including several premetastatic and proangiogenic genes (9-12). Several laboratories have demonstrated that $\mathrm{NF}-\kappa \mathrm{B}$ is constitutively activated in several types of cancers (13-18). However, the precise mechanism underscoring $\mathrm{NF}-\kappa \mathrm{B}$ activation in osteosarcoma cells remains to be elucidated.

It has been recently demonstrated that a newly identified type of polyubiquitin chain, a linear polyubiquitin chain, plays a critical role in NF- $\mathrm{NB}$ activation (19-21). Linear polyubiquitin chains are specifically generated by the linear ubiquitin chain assembly complex (LUBAC), which is composed of HOIL-1L, HOIP and SHARPIN. Upon stimulation with inflammatory cytokines, including TNF- $\alpha$ or IL- $1 \beta$, LUBAC specifically recognizes and conjugates linear polyubiquitin chains onto NEMO, which triggers the activation of IKK and subsequent $\mathrm{NF}-\kappa \mathrm{B}$ activation (19). A mutation in Sharpin causes chronic proliferative dermatitis (cpdm) in mice, which is characterized by chronic dermatitis, impaired secondary lymphoid organ development, and osteopenia (21-23). Moreover, we have observed that the induced expression of LUBAC enhances NF- $\kappa$ B activation (20). Although linear polyubiquitin-induced $N F-\kappa B$ activation is suggested to be involved in some diseases, there have been no reports directly describing the relationship between LUBACmediated NF- $\kappa \mathrm{B}$ activation and tumor progression. In the present study, we demonstrate that LUBAC-mediated NF- $\kappa$ B activation is involved in the lung metastasis of osteosarcoma cells.

\section{Materials and methods}

Cells and animals. We used a murine osteosarcoma cell line (Dunn cells), a highly metastatic subclone of Dunn cells (LM8), and a clone of LM8 cells that stably expresses luciferase (LC2LM8) provided by Dr Y. Yui from the Department of Biology at the Osaka Medical Center of Cancer and Cardiovascular Diseases (24). Cells were cultured in Dulbecco's modified Eagle's medium (DMEM) containing 10\% fetal bovine serum (FBS) (Life Technologies, Gaithersburg, MD, USA) in humidified air with $5 \% \mathrm{CO}_{2}$ at $37^{\circ} \mathrm{C}$. To measure cell growth rates, cells were seeded at a density of $2 \times 10^{4}$ cells per 6 -well dish. The number of cells was counted manually every 24 h. C3H female mice, aged 5-8 weeks, were purchased from Japan Oriental Yeast Co., Ltd. (Tokyo, Japan). All mice were housed under specific pathogen-free conditions with a 12-h light and dark cycle. The housing conditions and the experimental protocol were approved by the Animal Care and Use Committee of Osaka University.

Plasmids. The shRNA expression vector, pBluescriptKSH1-mHOIL-1L sh RNA was constructed by inserting mHOIL-1L shRNA between the HindIII and BamHI sites of the pBluescriptKS-H1 vector. The HOIL-1L shRNA-target sequence is 5'-gatccc-GCAGACGACAGAGATGCTAAAttcaagaga TTTAGCATCTCTGTCGTCTGCtttttggaaa-3'. pCMV-sport6ICAM-1 was purchased from Open Biosystems (Huntsville, AL, USA).
Transfection and generation of stable HOIL-1L knockdown clones. Transient transfection of LC2LM8 cells was performed by using Lipofectamine 2000 (Invitrogen, Carlsbad, CA, USA) according to the manufacturer's protocol. To establish HOIL-1L knockdown of LM8 cells, LM8 cells were transfected with pBluescriptKS-mHOIL-1L shRNA and pSV-Neo by using Lipofectamine 2000 according to the manufacturer's instructions. Stably transfected clones were establishing with G-418 selection. The level of HOIL-1L expression was verified by Western blotting with anti-HOIL-1L antibody.

Luciferase assay. Luciferase reporter plasmids pGL4.32-luc2PNF- $\kappa$ B-RE-Hygro and pGL4.74-hRuc-TK were purchased from Promega (Madison, WI, USA). Twenty-four hours after transfection with the appropriate plasmids, cells were lysed, and the luciferase activity was measured in a Lumat luminometer (Berthold, Germany) by using the Dual-Luciferase Reporter Assay Systems (Promega).

Assay of anchorage-independent growth. Soft agar assays were performed as follows: a bottom layer of $0.6 \%$ agar DMEM with $20 \%$ FBS was first placed onto 6 -well dishes. Then, $2 \times 10^{4}$ cells were seeded in $0.24 \%$ top agar containing DMEM with $20 \%$ FBS. The number of colonies that were $>200 \mu \mathrm{m}$ in diameter were counted in five $\mathrm{x} 40$ microscopic fields from three dishes after 7 days of cultivation.

Invasion assay. Invasion assays were performed by using modified Boyden chambers with a micropore membrane-coated Matrigel (BD Biosciences, Bedford, MA, USA). Cells in DMEM were added to the upper chamber, and the lower chamber was filled with DMEM containing 10\% (v/v) FBS. After incubation for $22 \mathrm{~h}$, non-invading cells on the upper surface were carefully removed with a cotton swab. The filters were then fixed, stained and photographed. Invasion was quantified by counting the invading cells in five random fields per filter by microscopy. Each clone was plated in duplicate for each experiment. We repeated the assay four times.

Gelatin zymography. For the evaluation of MMP-2 and -9 activity, gelatin zymography with a gelatin zymo-electrophoresis kit (Primary Cell, Hokkaido, Japan) was performed according to the manufacturer's directions. Conditioned media from each cell culture ( $1 \mathrm{ml} / 6-\mathrm{cm}$ dish) were centrifuged to remove insoluble material. Photographs were taken with a LAS3000 imaging system (Fujifilm, Tokyo, Japan).

Western blotting. Samples were separated by SDS-PAGE and transferred to PVDF membranes. After blocking in PBS containing $0.1 \%$ Tween-20 and 5\% skim milk, the membrane was incubated with the appropriate primary antibodies, followed by incubation with horseradish peroxidase-labeled anti-mouse or anti-rabbit IgG antibodies (GE Healthcare, Buckinghamshire, UK). The membrane was then visualized by using enhanced chemiluminescence and analyzed with a LAS3000 imaging system. Antibodies against HOIP, HOIL-1L, or SHARPIN have been described (25). Antibodies against ICAM-1 and $\beta$-actin were obtained from Santa Cruz Biotechnology (Santa Cruz, CA, USA). 
In vivo metastasis assay. Lung metastasis of LM8 cells and two HOIL-1L stable knockdown clones (Lu-1 and Lu-2) were evaluated as follows: a total of $10^{6} \mathrm{LM} 8$ cells, Lu- 1 cells or Lu- 2 cells were suspended in $100 \mu \mathrm{l}$ PBS and inoculated subcutaneously into the backs of mice. All mice were sacrificed on day 28 , and primary tumors and lungs were collected for histological studies. We measured the wet weight of the excised tumors and counted the number and measured the size of metastatic nodules in the lungs.

In vivo bioluminescence imaging. At $24 \mathrm{~h}$ after transfection with appropriate plasmids, $1 \times 10^{6}$ LC2LM8 cells suspended in $100 \mu 1$ PBS were injected into the lateral tail vein of mice. At 1 and $24 \mathrm{~h}$ after injection, we imaged mice with the IVIS LuminaII Imaging System (Caliper LifeSciences, Hopkinton, MA, USA). Mice were injected intraperitoneally with D-Luciferin (Caliper LifeSciences) $10 \mathrm{~min}$ prior to imaging. A region of interest (ROI) of the same size and shape that covered the entire thoracic cavity was applied to every image. Total flux (ps-1) in the ROI was measured. Data were analyzed by using IVIS Living Image software (Caliper LifeSciences). Seven days after tail vein injection, the mice were sacrificed, and the number of metastatic nodules in the lungs was evaluated.

Histological analyses. The tumors derived from implanted cells and the lungs were removed, fixed in $10 \%$ formalin, embedded in paraffin, and then cut into $3-\mu \mathrm{m}$ sections, followed by staining with hematoxylin and eosin. The lungs were evaluated microscopically to confirm the presence of lung metastasis.

Statistical methods. Results are presented as the average \pm standard deviation (SD) or as the average \pm standard error of the mean (SEM), as indicated in the figure legends. Between-group statistical significance was determined by using the Student's t-test or Fisher's exact test. A P $<0.05$ was considered statistically significant.

\section{Results}

Increased expression of LUBAC in highly metastatic LM8 cells. We have previously observed that NF- $\kappa \mathrm{B}$ is involved in the initial stage of lung metastasis of LM8 osteosarcoma cells (7) and that the induction of LUBAC components enhances NF- $\kappa \mathrm{B}$ activation (20). Therefore, we evaluated the expression levels of the LUBAC subunits (HOIP, HOIL-1L, and SHARPIN) in Dunn and LM8 cells to investigate the potential involvement of LUBAC-mediated NF- $\kappa \mathrm{B}$ activation in the lung metastasis of osteosarcoma cells. As shown in Fig. 1A, the amounts of all three subunits of LUBAC were increased in LM8 cells as compared to their parental Dunn cells. We then evaluated NF- $\kappa$ B activity in LM8 and Dunn cells with a luciferase reporter assay and found that the $N F-\kappa B$ activity of LM8 cells cultured in medium was higher than that of Dunn cells. LM8 cells, but not Dunn cells, are capable of spontaneously developing lung metastasis, although both cells form similarly sized tumor masses at the inoculation site in mice (5). Thus, these results suggest that increased LUBAC expression and $\mathrm{NF}-\kappa \mathrm{B}$ activity are involved in the spontaneous lung metastatic ability of LM8 cells.
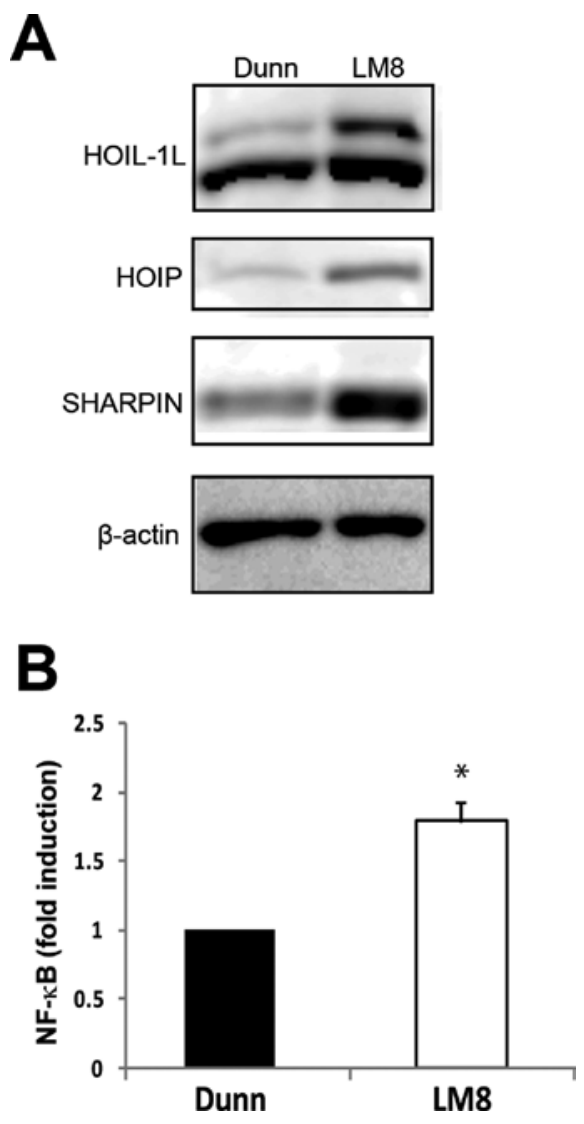

Figure 1. Correlation of LUBAC expression with NF- $\mathrm{BB}$ activity in Dunn and LM8 cells. (A) The expression of HOIL-1L/HOIP/SHARPIN was determined by Western blotting using total cell lysates. (B) Luciferase reporter assay for NF- $\mathrm{kB}$ transcriptional activity was performed as described in Materials and methods. Representative data from three different experiments. Columns, mean; bars, SD. *Significant difference by the Student's t-test.

Involvement of LUBAC-induced NF- $\mathrm{B}$ B activation in anchorageindependent growth and invasiveness of LM8 cells in vitro. To determine if the increased expression of LUBAC is involved in enhanced NF- $\kappa$ B activity in LM8 cells, we generated HOIL-1L knocked-down LM8 cells. As shown in Fig. 2A, the amount of HOIL-1L in the two stably knocked down clones, Lu-1 and Lu-2, was 17.5 and $10.8 \%$ of that in LM8 cells, respectively. We have previously shown that the amount of HOIP and SHARPIN was also decreased in HOIL-1L null cells, possibly because of the destabilization of the other two subunits of LUBAC in the absence of HOIL-1L $(20,21)$. As expected, the amount of HOIP and SHARPIN in Lu-1 and Lu-2 cells was significantly reduced as compared to that in LM8 cells (Fig. 2A), indicating that the knocked-down level of HOIL-1L in LM8 cells reduced the amount of LUBAC. We next examined the NF- $\kappa \mathrm{B}$ activity by using luciferase reporter assays and found that the $\mathrm{NF}-\kappa \mathrm{B}$ activity of Lu-1 and Lu-2 cells cultured in medium was significantly attenuated as compared to that of the LM8 cells (Fig. 2B).

Although the proliferation of Lu-1 and Lu-2 cells in DMEM supplemented with $10 \%$ FBS was not overtly affected (Fig. 2C), the number as well as the size of colonies formed in soft agar were significantly smaller in both Lu-1 and Lu-2 cells than in LM8 cells (Fig. 2D). These results indicate that the LUBAC-mediated NF- $\kappa \mathrm{B}$ activation pathway is involved in the anchorage-independent growth of LM8 cells . 
A
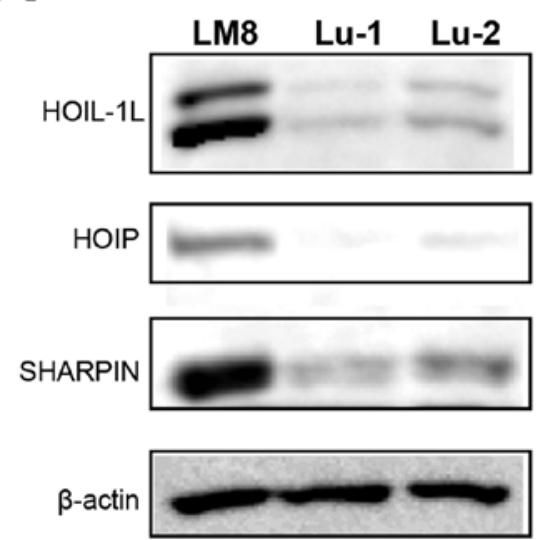

C

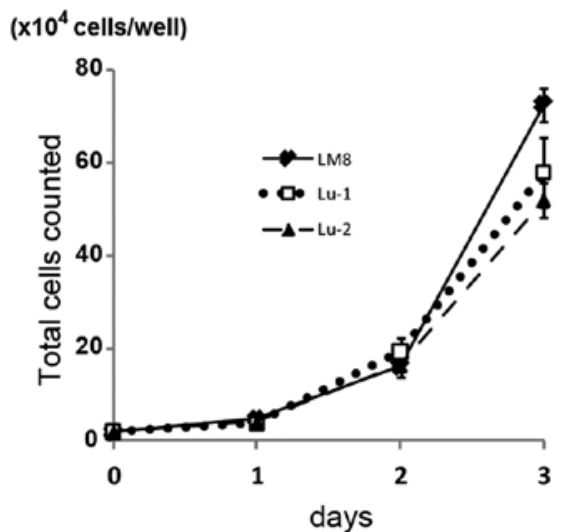

B

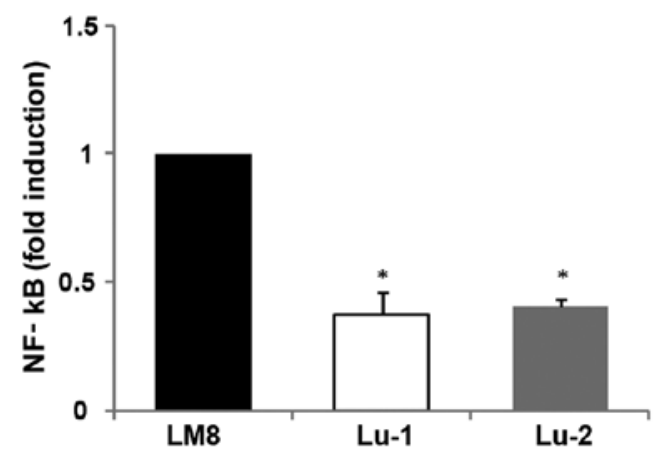

D

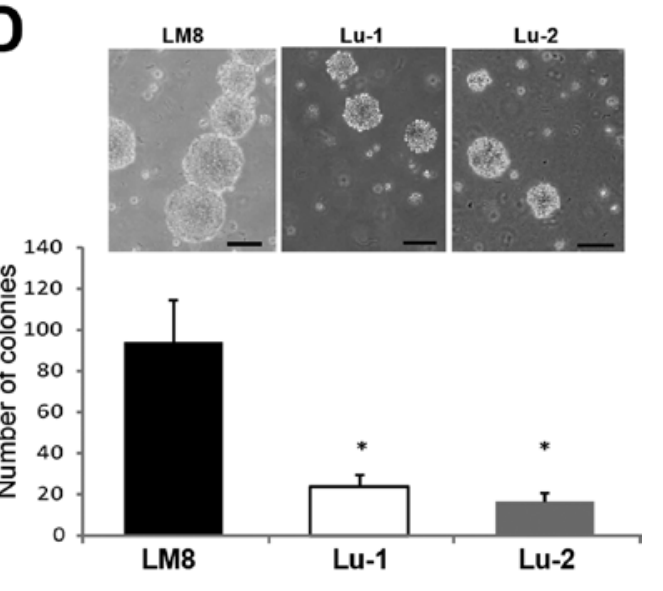

Figure 2. Stable suppression of LUBAC expression with the HOIL-1L siRNA expression vector. (A) The LM8 cells were stably transfected with a HOIL-1L siRNA expression vector. The expression of HOIL-1L/HOIP/SHARPIN was examined by Western blotting. (B) Luciferase reporter assay for NF- $\mathrm{B}$ transcriptional activity. Representative data from three different experiments. (C) Cell growth curves of LM8, Lu-1 and Lu-2 cells. The number of cells was counted every day. Representative data from three different experiments. (D) Anchorage-independent growth of LM8, Lu-1 and Lu-2 cells. The upper panel shows representative photos of soft agar colonies formed by LM8, Lu-1 and Lu-2 cells. Scale bar, $200 \mu \mathrm{m}$. The lower panel shows the mean number of colonies in five 440 microscopic fields from three different experiments. Columns, mean; bars, SD. "Significant differences compared with LM8 by the Student's t-test.

The cell invasiveness is one of the key characteristics that seem to be tightly associated with the metastatic potential of tumor cells. MMPs and ICAM-1 have been shown to be involved in cell invasiveness (26-31).

Gelatin zymography revealed reduced MMP2 activity in Lu-1 and Lu-2 cells, although MMP9 is not expressed in LM8 cells or their HOIL-1L-knockdown subclones (Fig. 3A and B). Similarly, Western blot analyses showed that ICAM-1 expression was decreased in Lu-1 and Lu-2 cells (Fig. 3C). To examine whether the reduced MMP2 activity and ICAM-1 expression of Lu-1 and Lu-2 cells contributed to the decreased invasiveness in extravasation, we performed a Matrigel invasion assay utilizing a Boyden chamber. Fig. 3D and E show that the numbers of Lu-1 and Lu-2 cells invading through the membrane-coated Matrigel were significantly less than that of control cells. Collectively, these results suggest that the LUBAC-mediated $\mathrm{NF}-\kappa \mathrm{B}$ activation pathway is involved in the invasiveness and anchorage-independent growth of LM8 cells.

Reduction of LUBAC attenuates the lung metastasis of LM8 cells. To determine if the attenuated LUBAC-mediated NF- $\mathrm{NB}$ activation activity affected the metastatic potential of LM8 cells, we next examined the metastatic behavior of LM8, Lu-1 and Lu-2 cells in the spontaneous metastatic model. Four weeks after inoculation of the cells into the backs of mice, we sacrificed the mice and examined metastatic lesions in their lungs and the weight of the primary tumors in their backs. Lung metastasis was observed in all mice $(15 / 15)$ inoculated with LM8 cells, whereas lung metastasis was found in $23 \%$ (4/17) or $0 \%(0 / 14)$ of mice inoculated with $\mathrm{Lu}-1$ or $\mathrm{Lu}-2$ cells, respectively. The representative histology of lungs of mice inoculated with LM8, Lu-1 or Lu-2 cells is shown in Fig. 4A. The average number of metastatic nodules per lung was $21.3 \pm 8.9,1.2 \pm 0.8$ or 0 in mice inoculated with LM8, Lu-1, or Lu-2 cells, respectively (Fig. 4B). Metastatic nodules of Lu-1 cells were smaller than those of LM8 cells (Fig. 4C). The primary tumor wet weight of mice inoculated with Lu-1 cells was significantly lower than that of mice inoculated with LM8 or Lu-2 cells (Fig. 4D). These data suggest that the LUBAC-mediated NF- $\kappa$ B activation pathway plays critical roles in the lung metastasis of LM8 cells.

Inhibition of LUBAC-mediated $N F-\kappa B$ activation decreases cell retention in the lungs. To elucidate the specific steps of the metastatic process to which LUBAC-mediated NF- $\kappa \mathrm{B}$ activa- 


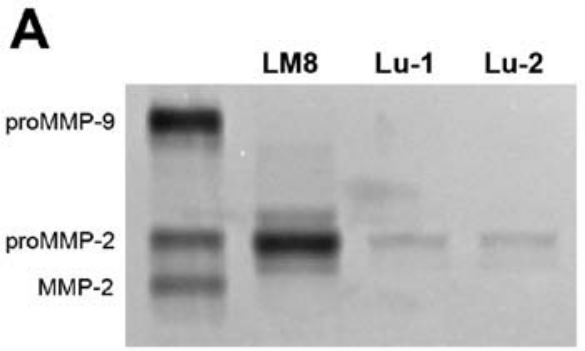

B

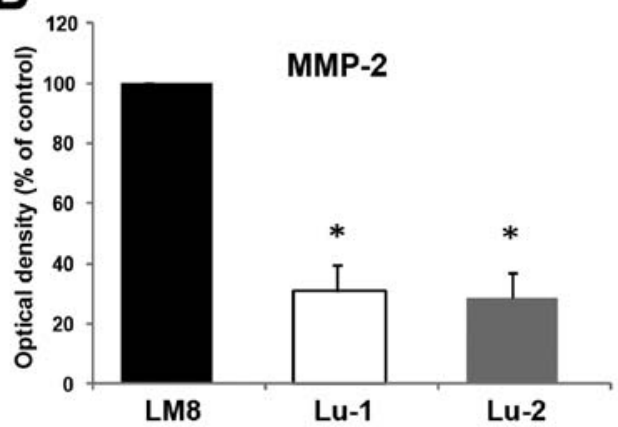

C

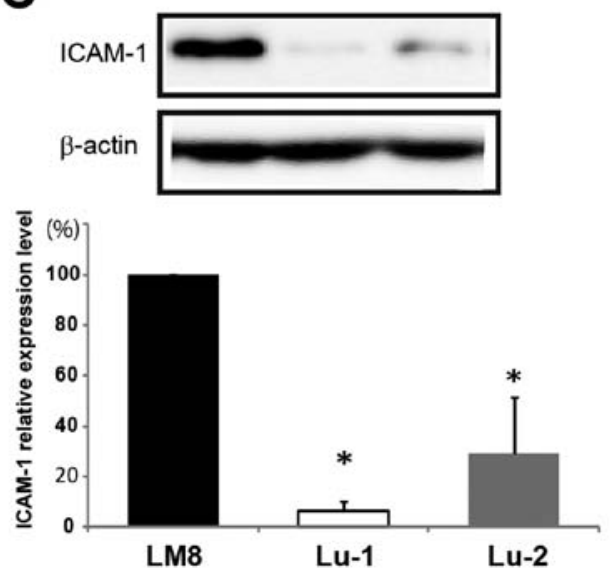

D

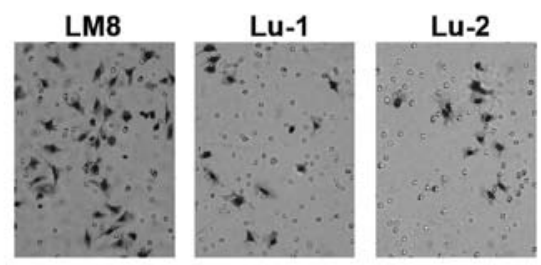

E

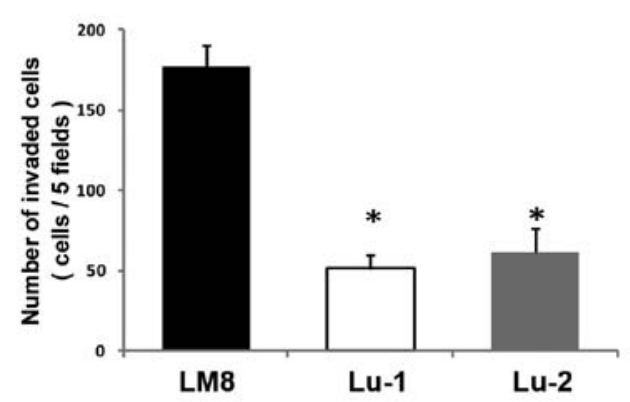

Figure 3. Suppression of LUBAC-induced NF- $\kappa$ B activation inhibits invasiveness of LM8 cells. (A) Gel zymography is shown. (B) Densitometric analysis of MMP-2 is shown. Representative data from three different experiments. (C) The upper panel shows a representative blot. The lower panel shows a quantitative analysis of relative ICAM-1 expression normalized by $\beta$-actin and calculated by densitometry ( $n=3)$. (D) Invasion assay. Representative cells invade through the Matrigel and membrane. (E) The invasion was quantified as described in Materials and methods. Columns, mean; bars, SD. *Significant differences as compared with LM8 by the Student's t-test. tion contributes, we examined the effect of decreased LUBAC function on the ability of tumor cells to extravasate into the lung.

To do so, we utilized LC2LM8 cells, a clone of LM8 cells that stably expresses luciferase. LC2LM8 cells transiently transfected with HOIL-1L siRNA expressing vector or control empty vector were injected into the tail vein. An in vivo imaging system was used to monitor the lungs 1 and $24 \mathrm{~h}$ after the injection. Western blot analyses confirmed that the expression levels of not only HOIL-1L but also HOIP and SHARPIN were reduced in HOIL-1L knocked-down cells as compared to control LC2LM8 cells (Fig. 5A). One hour after the inoculation of cells, no significant difference in the intensities of bioluminescence in the lung was observed between mice injected with LC2LM8 cells transfected with HOIL-1L siRNA and control vector. However, the intensity of bioluminescence in the lung was significantly reduced in mice injected with HOIL-1L siRNA LC2LM8 (siHOIL-1L) cells as compared with mice injected with control empty vector (control) LC2LM8 cells $24 \mathrm{~h}$ after inoculation (Fig. 5B and C). Seven days after inoculation, metastatic lesions in the lungs were assessed under a microscope. While many metastatic lesions were identified in the lungs of mice injected with control LC2LM8 cells (average,
$98 \pm 24$ per lung), only a few metastatic lesions were observed in the mice injected with siHOIL-1L LC2LM8 cells (average, $3 \pm 2$ nodules per lung) (Fig. 5D and E).

We then analyzed the mechanism underlying the strong inhibition of tumor cell retention to the lung by suppression of LUBAC-mediated NF- $\mathrm{NB}$ activation. We focused on ICAM-1 because the elevated expression of ICAM-1 has been observed in some cancers with distant metastases, and ICAM-1 is one of the target genes of NF- $\kappa \mathrm{B}$ (12). As expected, ICAM-1 expression in LC2LM8-HOIL-1L-silenced cells was lower than that in LC2LM8 control cells (Fig. 5A), suggesting the involvement of ICAM-1 in lung metastasis of LM8 cells. Since the transient introduction of ICAM-1 expression vector into LC2LM8-HOIL-1L siRNA cells transiently induced ICAM-1 expression (Fig. 5A), we then injected ICAM-1-expressing LC2LM8-HOIL-1L siRNA (siHOIL-1L+ICAM-1) cells into mice. One hour after injection into mice, siHOIL-1L+ICAM-1 cells could be observed in the lung region at a level comparable to siHOIL-1L and control LC2LM8 cells. However, in sharp contrast to siHOIL-1L LC2LM8 cells, a significant amount of siHOIL-1L+ICAM-1 LC2LM8 cells remained in the lungs, and the intensities of bioluminescence in the lungs of mice injected 


\section{A}

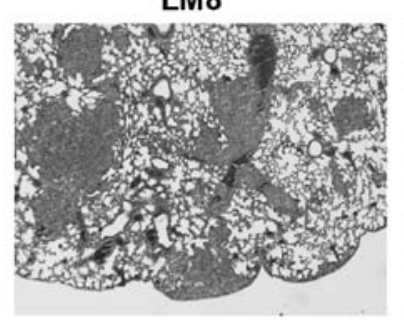

B

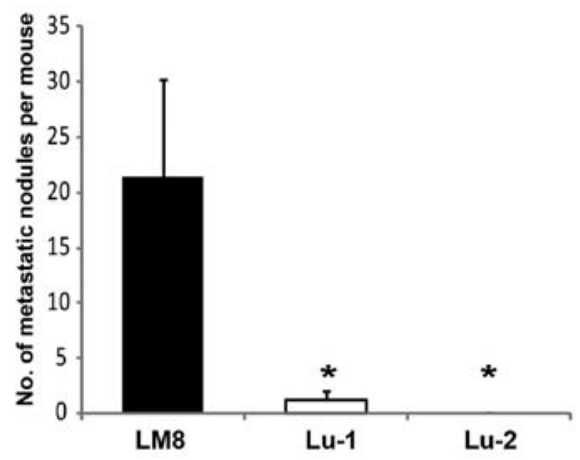

D

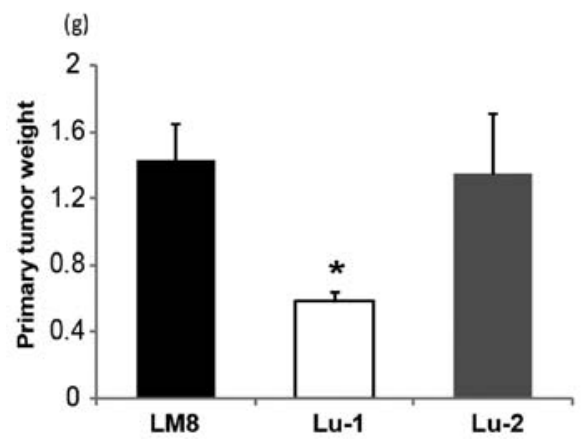

Lu-1

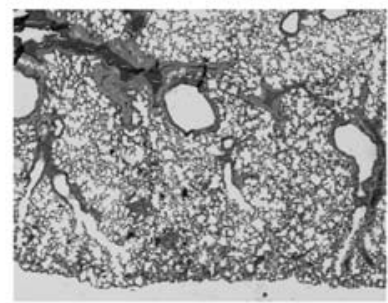

Lu-2

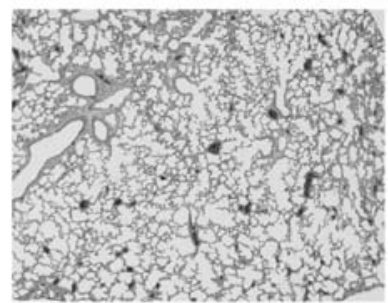

C

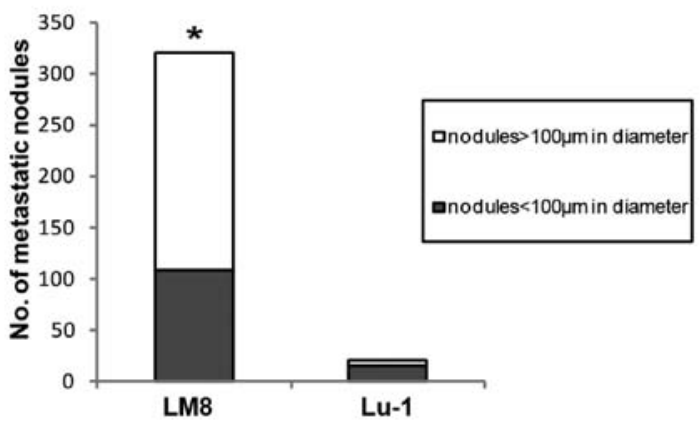

Figure 4. Reduction of LUBAC attenuates the lung metastasis of LM8 cells. (A) Representative H\&E-stained sections of lungs removed from each experimental group. (B) The total number of lung metastatic nodules in each individual mouse was counted under the microscope. Columns, mean; bars, SEM. "Significant difference as compared with LM8 by the Student's t-test. (C) Distribution of metastatic nodule size after LM8 or Lu-1 cell orthotopic implantation. Columns, total number of each group. "Significant difference by Fisher's exact test. (D) Primary tumor weight of each experimental group. Columns, mean; bars, SEM. "Significant difference compared with LM8 by the Student's t-test.

with siHOIL-1L+ICAM-1 LC2LM8 cells is comparable to that in mice injected with control LC2LM8 cells at $24 \mathrm{~h}$ after inoculation (Fig. 5B and C). The average number of metastatic nodules per lung was not significantly different between mice injected with siHOIL-1L+ICAM-1 LC2LM8 cells and mice injected with control LC2LM8 cells (Fig. 5D and E). Collectively, these results strongly suggest that enhanced expression of ICAM-1 is involved in the extravasation of LM8 osteosarcoma cells to the lungs, which results in the formation of metastatic nodules. This metastatic pathway appears to be at least partially provoked by the LUBAC-mediated NF- $\mathrm{NB}$ activation pathway.

\section{Discussion}

Lung metastasis of osteosarcomas is regarded to be an example of hematogenous metastasis. Hematogenous metastasis of malignant tumors is a multistep event. First, tumor cells are detached from primary lesions and migrate into the blood stream. Then, the cancer cells adhere to blood vessels of distant organs, extravasate and colonize to form a metastatic lesion. The capacity of malignant cells to adhere to the vascular endothelium in distant organs is an essential step in the formation of metastatic nodules. Various

adhesive molecules including ICAM-1 have been identified as responsible molecules for invasion, extravasation and metastasis (32). Clinical studies have shown that the expression level of ICAM-1 in malignant cells in patients correlates positively with primary tumor progression and the occurrence of metastasis (33-36). Moreover, recent studies showed that ICAM-1 on melanoma cells could promote anchoring of entrapped melanoma cells to the lung endothelium and the subsequent development of lung metastasis $(37,38)$. Inhibition of ICAM-1 function by siRNA or blocking antibodies has been reported to result in a strongly decreased metastatic potential $(39,40)$. The lung microenvironment is specific with many alveolar capillaries consisting of lung microvascular endothelial cells. Since the lung endothelial cells express lymphocyte function antigen-1 (LFA-1), which is a specific ligand for ICAM-1 (41), ICAM-1 is regarded to be one of the candidate molecules to induce adhesion and extravasation, which are initial steps in lung metastasis. Indeed, we have shown that ICAM-1 is involved in the initial steps of lung metastasis of LM8 cells by using an experimental metastasis model with intravenously inoculated tumor cells (Fig. 5).

It has been well established that ICAM-1 expression is induced by $\mathrm{NF}-\kappa \mathrm{B}$ (12). We have also shown that continu- 

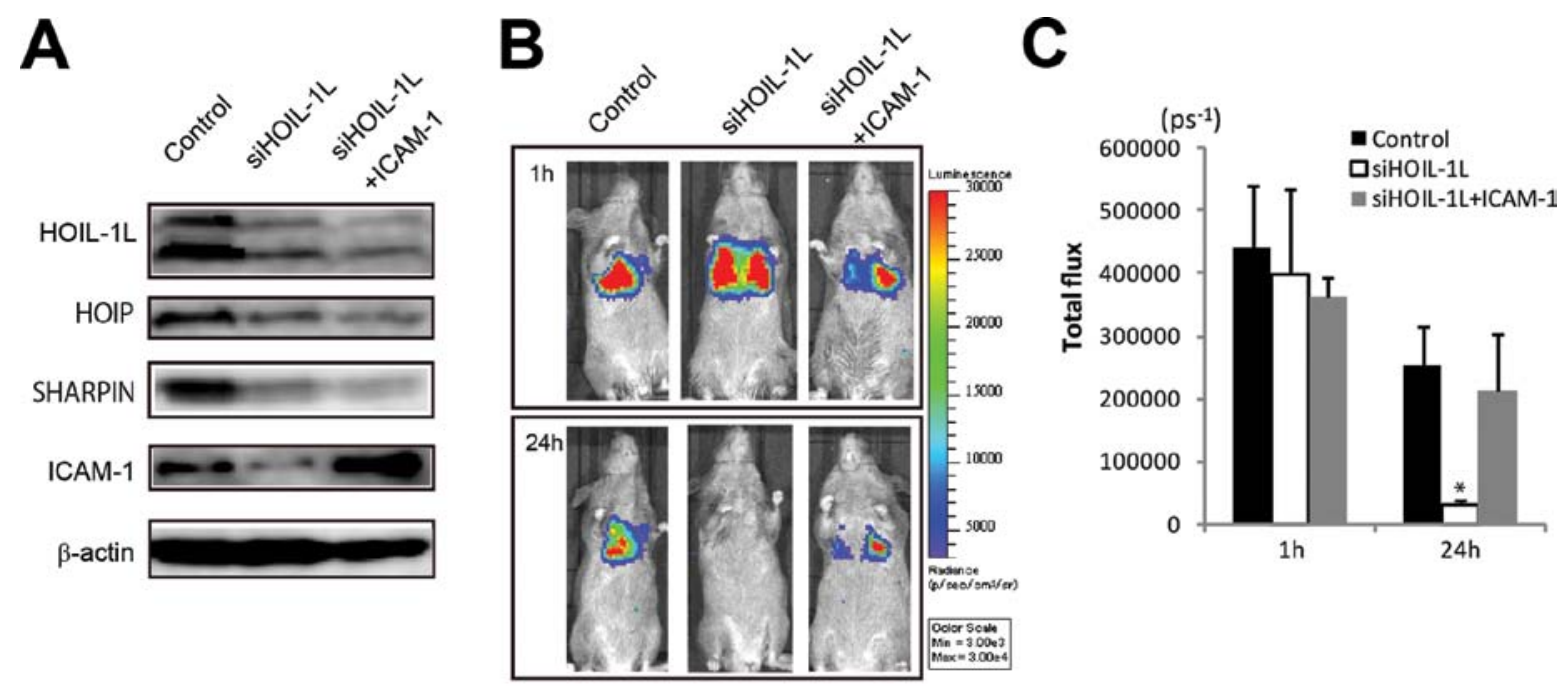

D

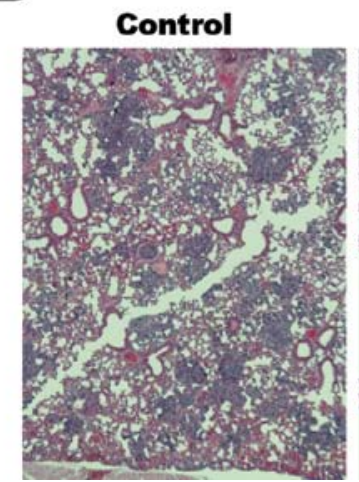

siHOIL-1L

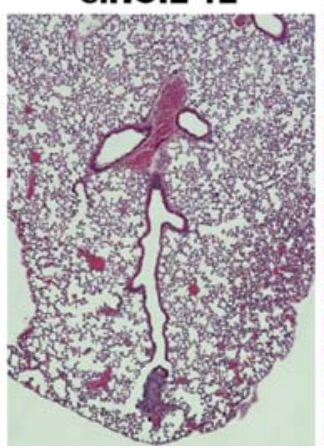

SIHOIL-1L+ICAM-1

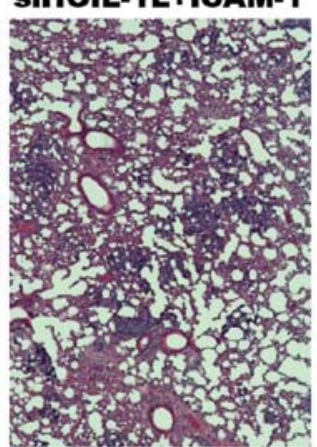

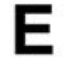

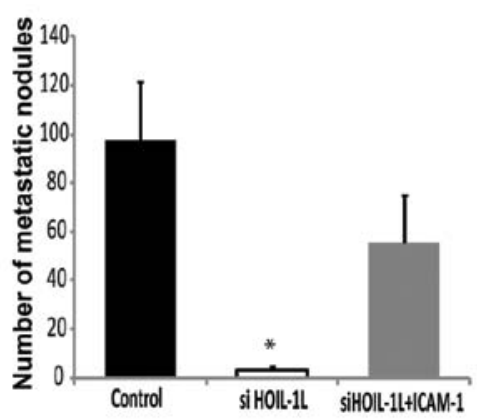

Figure 5. Inhibition of LUBAC-mediated NF- $\kappa$ B activation decreases cell retention in the lung through ICAM-1. (A) The expression of HOIL-1L and ICAM-1 was analyzed by Western blot analysis. (B) The representative bioluminescence imaging in vivo of control, siHOIL-1L and siHOIL-1L+ICAM-1 LC2LM8 at 1 and $24 \mathrm{~h}$ after intravenous inoculation. Bioluminescence is presented as a pseudoscale: red, highest photon flux; blue, lowest photon flux. Quantification of bioluminescence obtained from each group $(\mathrm{n}=10)$ is shown. (C) Quantification of bioluminescence imaging signal intensity in the three groups at 1 and $24 \mathrm{~h}$ after inoculation. Quantified values are shown in total flux. (D) Representative H\&E-stained sections of lungs at 7 days after inoculation. (E) Total numbers of lung metastatic nodules in individual mice were counted under the dissection scope. Columns, mean; bars, SD. *Significant differences as compared with the control group by the Student's t-test.

ously activated NF- $\kappa \mathrm{B}$ is related to the high incidence of lung metastasis of LM8 cells (6-8). LUBAC-induced linear polyubiquitination plays crucial roles in $\mathrm{NF}-\kappa \mathrm{B}$ activation, and induced expression of LUBAC augments NF- $\mathrm{BB}$ activation (19-21). We have observed that enforced expression of all three components of LUBAC is involved in the induction of ICAM-1 expression in LM8 cells, which leads to the generation of metastatic nodules in the lung, at least in part. In addition to a reduction of the number of metastatic nodules in the lung, the size of the metastatic nodules of Lu-1 cells, in which the expression of LUBAC was attenuated, was much smaller than those of control LM8 cells (Fig. 4B and C); this was also the case with LC2LM8 cells treated with siRNA for HOIL-1L or HOIL-1L and ICAM-1 (Fig. 5D and data not shown). These results suggest that the attenuation of NF- $\kappa \mathrm{B}$ activation by the suppression of LUBAC expression also plays a role in the growth of tumor cells in the lung after extravasation. We have demonstrated that the suppression of LUBAC in LM8 cells also reduced the anchorage-independent growth capacity of osteosarcoma cells. Since suppression of $\mathrm{NF}-\kappa \mathrm{B}$ activation by the introduction of $\mathrm{I} \kappa \mathrm{B} \alpha$ an inhibitor of $\mathrm{NF}-\kappa \mathrm{B}$ activation, to A549 cells suppressed both anchorage-independent growth and lung metastasis of the lung cancer cells (43), substantial suppression of NF- $\kappa \mathrm{B}$ may attenuate the growth of malignant cells in metastatic lesions in the lung. The observation that suppression of NF- $\kappa \mathrm{B}$ activation by $N F-\kappa B$ decoy oligonucleotide reduced the size of the metastatic nodules of LM8 cells in the lung is consistent with our results (7). It has been recently shown that the introduction of SHARPIN, a component of LUBAC, enhanced the anchorageindependent growth of $\mathrm{CHO}$ cells, although the amounts of the other components of LUBAC were not determined (42). Collectively, LUBAC-induced NF- $\mathrm{B}$ activation might be involved not only in the attachment of LM8 cells to the lung, but also in the other aspects of metastasis after extravasation, such as invasion activity and/or anoikis resistance, in our experimental model.

We have observed that the expression of all three components of LUBAC was augmented in LM8 cells, which plays crucial roles for lung metastasis of the sarcomas. Our extensive attempts to establish Dunn cells that overexpress all three components of LUBAC by introducing expression vector cDNAs have been unsuccessful (data not shown). Moreover, 
the agents that induce the expression of LUBAC components have not been identified. Thus, the mechanism underlying the enhanced expression of LUBAC components in LM8 cells remains unknown. We speculate that excessive overexpression of LUBAC is toxic for Dunn cells and that LM8 cells could acquire the ability to allow LUBAC overexpression during their establishment. It is possible that the overexpression of LUBAC results in constitutively activated NF- $\mathrm{KB}$, which contributes to the highly metastatic potential of osteosarcoma cells. Therefore, it will be interesting to elucidate how the overexpression of LUBAC is regulated in LM8 cells and to examine whether there are correlations between the expression of LUBAC and the metastasis of malignant tumors, including osteosarcoma, in future clinical studies.

In conclusion, we show here that transcriptional activity of NF- $\mathrm{KB}$ is regulated by LUBAC in osteosarcoma cells. Our analysis demonstrates that a reduction in LUBAC drastically suppresses the metastatic abilities of osteosarcoma cells including cell invasiveness and lung metastasis. We have provided the first experimental evidence indicating that the activation of NF- $\mathrm{KB}$ by LUBAC could exert a biological effect in an animal model of human malignancy. We have previously shown that TNF- $\alpha-$ induced activation of JNK was not overtly impaired in embryonic fibroblasts (MEFs) from mice lacking HOIL-1L or SHARPINnull cpdm mice, although TNF- $\alpha$-induced NF- $\mathrm{KB}$ activation was severely impaired in MEFs from those mice $(19,20)$. These results suggested that the suppression of LUBAC activity mainly attenuates NF- $\mathrm{KB}$ activation without overtly affecting other signaling pathways. Therefore, LUBAC could provide a new therapeutic approach to treat this most common malignant bone tumor threatening the lives of children and young adolescents.

\section{Acknowledgements}

The authors are grateful to Yoshihiro Yui, Kiyoko Yoshioka and Kazuyuki Itoh for providing the LC2LM8 cells and their valuable discussions (Department of Biology, Osaka Medical Center of Cancer and Cardiovascular Diseases) and to Kiyoko Kamei, Mizuki Nakata and Mari Shinkawa for their excellent technical help in this study. This research was supported by grants from the Japan Society for the Promotion of Science (20591754, 23592184).

\section{References}

1. Goorin AM, Schwartzentruber DJ, Devidas M, et al: Presurgical chemotherapy compared with immediate surgery and adjuvant chemotherapy for nonmetastatic osteosarcoma: Pediatric Oncology Group Study POG-8651. J Clin Oncol 21: 1574-1580, 2003.

2. Ferrari S, Smeland S, Mercuri M, et al: Neoadjuvant chemotherapy with high-dose Ifosfamide, high-dose methotrexate, cisplatin, and doxorubicin for patients with localized osteosarcoma of the extremity: a joint study by the Italian and Scandinavian Sarcoma Groups. J Clin Oncol 23: 8845-8852, 2005.

3. Bacci G, Rocca M, Salone M, et al: High grade osteosarcoma of the extremities with lung metastases at presentation: treatment with neoadjuvant chemotherapy and simultaneous resection of primary and metastatic lesions. J Surg Oncol 98: 415-420, 2008.

4. Kager L, Zoubek A, Potschger U, et al: Primary metastatic osteosarcoma: presentation and outcome of patients treated on neoadjuvant Cooperative Osteosarcoma Study Group protocols. J Clin Oncol 21: 2011-2018, 2003.

5. Asai T, Ueda T, Itoh $\mathrm{K}$, et al: Establishment and characterization of a murine osteosarcoma cell line (LM8) with high metastatic potential to the lung. Int J Cancer 76: 418-422, 1998.
6. Asai T, Tomita Y, Nakatsuka S, et al: VCP (p97) regulates NFkappaB signaling pathway, which is important for metastasis of osteosarcoma cell line. Jpn J Cancer Res 93: 296-304, 2002.

7. Nishimura A, Akeda K, Matsubara T, et al: Transfection of NF-kappa B decoy oligodeoxynucleotide suppresses pulmonary metastasis by murine osteosarcoma. Gene Ther 18: 250-259, 2011.

8. Kishida Y, Yoshikawa $\mathrm{H}$ and Myoui A: Parthenolide, a natural inhibitor of Nuclear Factor-kappaB, inhibits lung colonization of murine osteosarcoma cells. Clin Cancer Res 13: 59-67, 2007.

9. Pahl HL: Activators and target genes of Rel/NF-kappaB transcription factors. Oncogene 18: 6853-6866, 1999.

10. Philip S, Bulbule A and Kundu GC: Osteopontin stimulates tumor growth and activation of promatrix metalloproteinase-2 through nuclear factor-kappa B-mediated induction of membrane type 1 matrix metalloproteinase in murine melanoma cells. J Biol Chem 276: 44926-44935, 2001.

11. Chilov D, Kukk E, Taira S, et al: Genomic organization of human and mouse genes for vascular endothelial growth factor C. J Biol Chem 272: 25176-25183, 1997.

12. Caldenhoven E, Coffer P, Yuan J, et al: Stimulation of the human intercellular adhesion molecule- 1 promoter by interleukin- 6 and interferon-gamma involves binding of distinct factors to a palindromic response element. J Biol Chem 269: 21146-21154, 1994.

13. Lenz G, Davis RE, Ngo VN, et al: Oncogenic CARD11 mutations in human diffuse large B cell lymphoma. Science 319: 1676-1679, 2008.

14. Wilson W III and Baldwin AS: Maintenance of constitutive IkappaB kinase activity by glycogen synthase kinase-3alpha/beta in pancreatic cancer. Cancer Res 68: 8156-8163, 2008.

15. Tai DI, Tsai SL, Chang YH, et al: Constitutive activation of nuclear factor kappaB in hepatocellular carcinoma. Cancer 89: 2274-2281, 2000.

16. Kato T Jr, Gotoh Y, Hoffmann A and Ono Y: Negative regulation of constitutive NF-kappaB and JNK signaling by PKN1-mediated phosphorylation of TRAF1. Genes Cells 13: 509-520, 2008.

17. Dhawan P, Su Y, Thu YM, et al: The lymphotoxin-beta receptor is an upstream activator of NF-kappaB-mediated transcription in melanoma cells. J Biol Chem 283: 15399-15408, 2008

18. Pianetti S, Arsura M, Romieu-Mourez R, Coffey RJ and Sonenshein GE: Her-2/neu overexpression induces NF-kappaB via a PI3-kinase/Akt pathway involving calpain-mediated degradation of IkappaB-alpha that can be inhibited by the tumor suppressor PTEN. Oncogene 20: 1287-1299, 2001.

19. Tokunaga F, Sakata S, Saeki Y, et al: Involvement of linear polyubiquitylation of NEMO in NF-kappaB activation. Nat Cell Biol 11: 123-132, 2009.

20. Tokunaga F, Nakagawa T, Nakahara M, et al: SHARPIN is a component of the NF-kappaB-activating linear ubiquitin chain assembly complex. Nature 471: 633-636, 2011.

21. Gerlach B, Cordier SM, Schmukle AC, et al: Linear ubiquitination prevents inflammation and regulates immune signalling. Nature 471: 591-596, 2011.

22. Xia T, Liang Y, Ma J, Li M, Gong M and Yu X: Loss-of-function of SHARPIN causes an osteopenic phenotype in mice. Endocrine 39: 104-112, 2011.

23. Liang Y, Seymour RE and Sundberg JP: Inhibition of NF-kappaB signaling retards eosinophilic dermatitis in SHARPIN-deficient mice. J Invest Dermatol 131: 141-149, 2011.

24. Yui Y, Itoh K, Yoshioka K, et al: Mesenchymal mode of migration participates in pulmonary metastasis of mouse osteosarcoma LM8. Clin Exp Metastasis 27: 619-630, 2010.

25. Kirisako T, Kamei K, Murata S, et al: A ubiquitin ligase complex assembles linear polyubiquitin chains. EMBO J 25: 4877-4887, 2006.

26. Radjabi AR, Sawada K, Jagadeeswaran S, et al: Thrombin induces tumor invasion through the induction and association of matrix metalloproteinase-9 and beta1-integrin on the cell surface. J Biol Chem 283: 2822-2834, 2008.

27. Kahari VM and Saarialho-Kere U: Matrix metalloproteinases and their inhibitors in tumour growth and invasion. Ann Med 31: 34-45, 1999.

28. Shima I, Sasaguri Y, Kusukawa J, et al: Production of matrix metalloproteinase- 2 and metalloproteinase- 3 related to malignant behavior of esophageal carcinoma. A clinicopathologic study. Cancer 70: 2747-2753, 1992.

29. Pazzaglia L, Ponticelli F, Magagnoli G, et al: Activation of metalloproteinases- 2 and -9 by interleukin-1alpha in S100A4-positive liposarcoma cell line: correlation with cell invasiveness. Anticancer Res 24: 967-972, 2004. 
30. Huang WC, Chan ST, Yang TL, Tzeng CC and Chen CC: Inhibition of ICAM-1 gene expression, monocyte adhesion and cancer cell invasion by targeting IKK complex: molecular and functional study of novel alpha-methylene-gamma-butyrolactone derivatives. Carcinogenesis 25: 1925-1934, 2004.

31. Lin YC, Shun CT, Wu MS and Chen CC: A novel anticancer effect of thalidomide: inhibition of intercellular adhesion molecule1-mediated cell invasion and metastasis through suppression of nuclear factor-kappaB. Clin Cancer Res 12: 7165-7173, 2006.

32. Kobayashi H, Boelte KC and Lin PC: Endothelial cell adhesion molecules and cancer progression. Curr Med Chem 14: 377-386, 2007.

33. Christiansen I, Gidlof C, Wallgren AC, Simonsson B and Totterman TH: Serum levels of soluble intercellular adhesion molecule 1 are increased in chronic B-lymphocytic leukemia and correlate with clinical stage and prognostic markers. Blood 84: 3010-3016, 1994.

34. Harning R, Mainolfi E, Bystryn JC, Henn M, Merluzzi VJ and Rothlein R: Serum levels of circulating intercellular adhesion molecule 1 in human malignant melanoma. Cancer Res 51: 5003-5005, 1991.

35. Toiyama Y, Miki C, Inoue Y, et al: Soluble intercellular adhesion molecule-1 as a prognostic marker for stage II colorectal cancer patients. Ann Surg Oncol 15: 1617-1624, 2008.
36. Maruo Y, Gochi A, Kaihara A, et al: ICAM-1 expression and the soluble ICAM-1 level for evaluating the metastatic potential of gastric cancer. Int J Cancer 100: 486-490, 2002.

37. Liang S, Fu C, Wagner D, et al: Two-dimensional kinetics of beta 2 -integrin and ICAM-1 bindings between neutrophils and melanoma cells in a shear flow. Am J Physiol Cell Physiol 294: C743-C753, 2008.

38. Huh SJ,Liang S, Sharma A, Dong C and Robertson GP: Transiently entrapped circulating tumor cells interact with neutrophils to facilitate lung metastasis development. Cancer Res 70: 6071-6082, 2010.

39. Rosette C, Roth RB, Oeth P, et al: Role of ICAM1 in invasion of human breast cancer cells. Carcinogenesis 26: 943-950, 2005

40. Yang YM, Du GJ and Lin HH: Experimental study of thalidomide for treatment of murine hepatocellular carcinoma. Di Yi Jun Yi Da Xue Xue Bao 25: 925-928, 2005.

41. Ten Kate M,Hofland LJ, van Grevenstein WMU, van Koetsveld PV, Jeekel $\mathbf{J}$ and van Eijck CHJ: Influence of proinflammatory cytokines on the adhesion of human colon carcinoma cells to lung microvascular endothelium. Int J Cancer 112: 943-950, 2004.

42. Jung J, Kim JM, Park B, et al: Newly identified tumor-associated role of human Sharpin. Mol Cell Biochem 340: 161-167, 2010. 